OPEN ACCESS

Edited by:

Pasquale Striano,

University of Genoa, Italy

Reviewed by:

Janet K. Kern,

Independent Researcher, Silver

Spring, United States

Marco Carotenuto,

University of Campania Luigi

Vanvitelli, Italy

Alberto Verrotti,

University of L'Aquila, Italy

*Correspondence:

Ying Han

hanying1568@126.com

Specialty section:

This article was submitted to

Pediatric Neurology,

a section of the journal

Frontiers in Neurology

Received: 07 August 2019 Accepted: 26 September 2019

Published: 05 November 2019

Citation:

Niu M, Li Q, Zhang J, Wen F, Dang W, Duan G, Li H, Ruan W, Yang P,

Guan C, Tian H, Gao X, Zhang S,

Yuan $F$ and Han Y (2019)

Characterization of Intestinal

Microbiota and Probiotics Treatment in Children With Autism Spectrum

Disorders in China.

Front. Neurol. 10:1084. doi: 10.3389/fneur.2019.01084

\section{Characterization of Intestinal Microbiota and Probiotics Treatment in Children With Autism Spectrum Disorders in China}

\author{
Manman Niu ${ }^{1,2}$, Qinrui Li ${ }^{1,3}$, Jishui Zhang ${ }^{4}$, Fang Wen ${ }^{4}$, Weili Dang ${ }^{5}$, Guiqin Duan ${ }^{6}$, \\ Haifeng $\mathrm{Li}^{7}$, Wencong Ruan ${ }^{7}$, Pingri Yang ${ }^{7}$, Chunrong Guan ${ }^{8}$, Huiling Tian ${ }^{9}$, \\ Xiaoqing Gao ${ }^{9}$, Shaobin Zhang ${ }^{10}$, Fangfang Yuan ${ }^{11}$ and Ying Han ${ }^{1 *}$ \\ ${ }^{1}$ Department of Pediatrics, Peking University First Hospital, Beijing, China, ${ }^{2}$ Department of Pediatrics, Beijing Tiantan \\ Hospital, Capital Medical University, Beijing, China, ${ }^{3}$ Department of Pediatrics, Peking University People's Hospital, Beijing, \\ China, ${ }^{4}$ National Center for Children's Health, Beijing Children's Hospital, Capital Medical University, Beijing, China, \\ ${ }^{5}$ Children's Encephalopathy Diagnosis and Rehabilitation Center, The First Affiliated Hospital of Henan University of Chinese \\ Medicine, Zhengzhou, China, ${ }^{6}$ Center of Children Psychology and Behavior, Henan Maternal and Child Health Hospital, \\ Zhengzhou, China, ${ }^{7}$ The Children's Hospital Zhejiang University School of Medicine, Hangzhou, China, ${ }^{8}$ Department of \\ Pediatric Rehabilitation, Jiningshi Renchengqu Women's and Children's Health Care Hospital, Jining, China, ${ }^{9}$ Department of \\ Pediatric Rehabilitation, Linyishi Women's and Children's Hospital, Linyi, China, ${ }^{10}$ Beijing Gutgene Technology Co. Ltd, \\ Beijing, China, ${ }^{11}$ Xinxiang Central Hospital, Xinxiang, China
}

Background: Most previous studies have found that human intestinal microbiota affect the symptoms of autism spectrum disorder (ASD), especially gastrointestinal (Gl) symptoms, but regarding this, there is limited data of non-western ethnicity. Probiotics can reconstitute the host intestinal microbiota and strengthen gastrointestinal function, however, clinical data proving the effect of probiotics treatment on ASD is lacking.

Methods: This study explored the significant differences between ASD and neurotypical (NT), and the improvement of applied behavior analysis (ABA) training in combination with probiotics, vs. ABA training only.

Results: We found significant differences between the ASD group and the NT group in the evenness of the intestinal microbiota and the relative abundance of the bacterial phyla and genus. At the phylum level, relative abundance of Bacteroidetes in the ASD group was significantly lower than in the NT group. At the genus level, the relative abundance of Bacteroides, Bifidobacterium, Ruminococcus, Roseburia, and Blautia in the ASD group was significantly lower than that in the NT group. After a 4-week ABA training program in combination with probiotics treatment, the ATEC and Gl scores decreased more than the control group with ABA training only.

Conclusion: Our findings suggest that intestinal microbiota is different between the NT children and the ASD children with or without Gl problems. In combination with ABA training, probiotics treatment can bring more benefit to ASD children. Clinical trials with a more rigorous design and larger sample size are indispensable for further validation.

Keywords: autism spectrum disorder, intestinal microbiota, probiotics treatment, children, China 


\section{INTRODUCTION}

Autism spectrum disorder (ASD) refers to a group of neurodevelopmental disorders with multiple, heterogenous causes. ASD is characterized by stereotyped behavior, language, and social interaction disorders. The incidence of ASD in children from 1.6 to 8 years old in China is approximately $39.23 / 10,000$ (1). The etiology of ASD is complex; both heredity and the environmental are involved (2). Studies have found that human intestinal microbiota affect the human brain and may affect human behavior and mental health through the gut-brain axis (3-7). The most common non-neurological indications in children with ASD are gastrointestinal symptoms (GI) such as constipation and diarrhea (8). Many studies have shown that the intestinal microbiota of autistic children with GI is different from that of neurotypical (NT) children (9-12), and the GI in the children with ASD corrects with their autistic manifestations (13).

Currently there is no cure for ASD. Children with ASD require long-term applied behavior analysis (ABA) training to improve their symptoms. Probiotics treatment can reconstitute the host intestinal microbiota, restore microbiota homeostasis, and strengthen gastrointestinal function. Animal models (14) and preliminary clinical trials have shown that probiotics treatment can alleviate ASD symptoms (15-17). Probiotics treatment may be a suitable therapy for ASD (18-20). Most previous studies of the relationship between ASD and GI have been conducted on participants of Western countries; research on children with ASD in China is scarce $(21,22)$. Therefore, this study aims to study the intestinal microbiota of children with ASD in China, and both ASD children with and without GI are included. Children with ASD are treated with probiotics and the clinical outcomes are investigated.

\section{MATERIALS AND METHODS}

\section{Subject Recruitment and Sample Collection}

The ethics committee of Peking University has reviewed and approved this study. It has been registered in the Chinese Clinical Trial Registry (ChiCTR1900023609) and all patients have signed the written informed consent. A total of 114 children with ASD (diagnosed according to Diagnostic and Statistical Manual of Mental Disorders, 5th Edition) (23) were recruited from hospital pediatric departments, hospital neurodevelopmental departments, and autistic rehabilitation institutions in Beijing, Shandong, Henan, and Zhejiang. In the meantime, a total of 40 neurotypical (NT) children from regular preschools were selected as the negative control group. ASD enrollment criteria were as follows: (1) diagnosis of ASD according to DSM-V; (2) age 3-8 years old; (3) no kinship between participants; (4) no antibiotics, probiotics, or other gastrointestinal treatments within 1 month prior to enrollment. ASD exclusion criteria were as follows: (1) No diagnosis of ADHD or other non-ASD neurological peculiarity, which cause autism-like manifestations; (2) children with other mental illnesses, brain organic diseases, severe liver and kidney diseases, and cardiovascular diseases; (3) children's guardians did not give permission for participation or did not complete follow-up. NT children were selected using the following criteria: (1) age 3-8 years old, normal development; (2) no family history of ASD; (3) children's guardians agreed to participate in the study and signed informed consent; (4) no antibiotics, or other gastrointestinal treatments within 1 month before enrollment.

First defecations of the day were collected at home by their parents. The DNA samples were extracted for PCR using OMEGA E.Z.N.A Stool DNA Kit and were quantified by Nanodrop. PCR was carried out on GeneAmp ${ }^{\circledR} 9700$ with Phusion High-Fidelity PCR Master Mix, the primers were $341 \mathrm{~F}$ and 806R.The mixture of purified PCR products was generated for Next-generation sequencing (NGS) library using Agencourt AMPure XP $60 \mathrm{ml}$ Kit following manufacturer's recommendations. The library quality was quantified by Qubit dsDNA HS Assay Kitwith the Qubit 2.0 fluorometer system. The multiplexed amplicons were sequenced using the Illumina MiSeq platform to generate 300 bp paired-end reads. De novo operational taxonomic unit picking was done using QIIME2 software (24).

\section{Probiotics Treatment}

Thirty-seven children with ASD were treated with 4-week applied behavior analysis $(\mathrm{ABA})$ training in combination with probiotics. Probiotics are lyophilized, water-soluble powder that contains 6 strains of bacteria; each strain has 1 billion CFU/gram. The dosage is $6 \mathrm{~g}$ per day (36 billion $\mathrm{CFU}$ in total). Twenty-eight other children with ASD were randomly selected as a control group and treated with ABA training alone. During the study, both groups were prohibited from the use of antibiotics, other probiotics, prebiotics, or any other treatment that might alter the intestinal microbiota.

\section{Evaluation of ASD-Related and Gastrointestinal Symptoms}

ASD-related symptoms were assessed using the Autism Treatment Evaluation Checklist (ATEC) before and after probiotics treatment. Clinical assessment information for children with ASD was provided by parents and reviewed by pediatric experts. ATEC and a questionnaire were used for follow-up. The questionnaire asked about GI, diet, sleep, mood, and behavior. GI was scored by parents. The criteria were as follows: 0 points for no GI abnormalities, 1 point for occasional abnormalities (1-2 episodes of diarrhea or only 1 bowel movement over 2 days per week), 2 points for frequent abnormalities (3-5 episodes of diarrhea or 1 bowel movement over 2 days per week), and 3 points for long-term or severe abnormalities (more than 5 episodes of diarrhea or 1 bowel movement over 5 days per week).

\section{Statistical Analysis}

Wilcoxon signed-rank test, paired wilcoxon test, and KruskalWallis test analysis were performed using $\mathrm{R}$ [http://cran.rproject.org/]. All $p$-values reported in the study were from two-tailed tests and $p$-values lower than 0.05 were accepted as significant in clinical data analysis. All $p$-values for bacterial 
microbiome analyses were corrected using the BenjaminiHochberg false discovery rate (FDR) correction, and the resulting corrected values were referred to as $q$-values. $q$-values lower than 0.05 were accepted as significant.

\section{RESULTS}

We here investigated 114 children with ASD and 40 NT children from 4 clinical centers. The average age of the participants was 4 years old (range: $3-8$ ). The ratio of male to female was $1: 1$ in the NT group and 5:1 in the ASD group. There were no GI abnormalities in the NT group. In the ASD group, $61.4 \%$ (70/114) of the patients had GI events, with the most common

TABLE 1 | Characteristics of study participants.

\begin{tabular}{lcc}
\hline & ASD & NT \\
\hline Subjects & $114 \mathrm{t}$ & 40 \\
Age (mean) & 4.5 & 4.2 \\
Gender & & \\
Female & 19 & 20 \\
Male & 95 & 20 \\
Gl & 70 & 0 \\
Constipation & 56 & 0 \\
Diarrhea & 7 & 0 \\
other & 7 & 0 \\
Non-Gl & 44 & 33
\end{tabular}

Data expressed as means with ranges when applicable. ASD, autism spectrum disorders subjects; NT, neurotypical subjects; NA, not applicable; ATEC, Autism treatment evaluation checklist. being constipation, affecting $80 \%$ of participants in that group (56/70) (Table 1).

We found significant differences between the ASD group and the NT group in the evenness of the intestinal microbiota and the relative abundance of the bacterial phyla and genus. The beta microbiota diversity index analysis did not yield significant results. In the $\alpha$ diversity analysis, the Shannon index of the ASD group with and without GI events was significantly higher than that of the NT group. However, the Simpson index of the ASD group with GI events was significantly lower than that of the NT group and the ASD without GI events, and no significant difference was observed between the NT group and the ASD without GI. The Simpson index is sensitive to microbiota evenness while the Shannon index is sensitive to the abundance of bacteria. Our results indicate that the ASD children in China had a higher intestinal microbiota abundance but a lower evenness (Figure 1). The ASD group without GI events showed a greater microbiota abundance than in the NT group and evenness similar to the NT group. At the phylum level, the relative abundance of Bacteroidetes and Actinobacteria in the ASD group was significantly lower than in the NT group, while the relative abundance of Proteobacteria in ASD group was higher than in the NT group. The Firmicutes in the ASD group was significantly greater than in the NT group. This result is consistent with results from previous research (9). At the genus level, the relative abundance of Bacteroides, Bifidobacterium, Ruminococcus, Roseburia, and Blautia in the ASD group was significantly lower than that in the NT group. However, Lachnospira was significantly more abundant than in the NT group (Table 2 ).

We conducted a 4-week ABA training program in combination with probiotics treatment for 37 children with ASD, among whom 22 had GI events and 15 did not. Our results
A Gl status NT ASD GI ASD nonGl

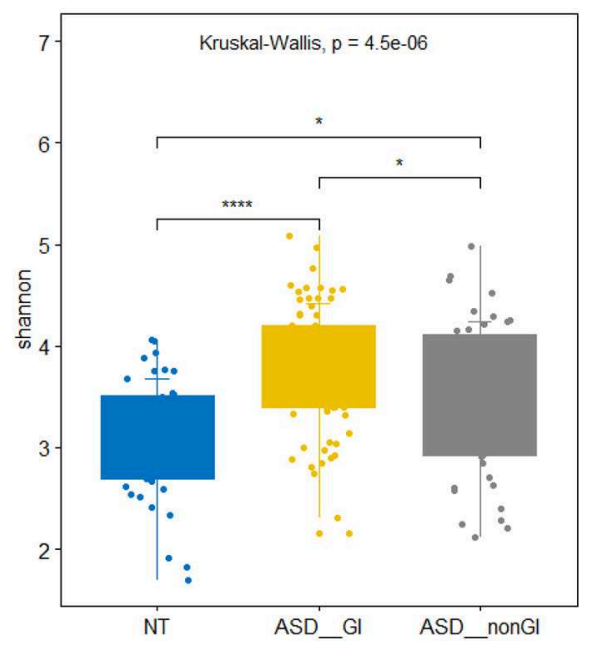

B Gl status NT ASD Gl ASD nonG

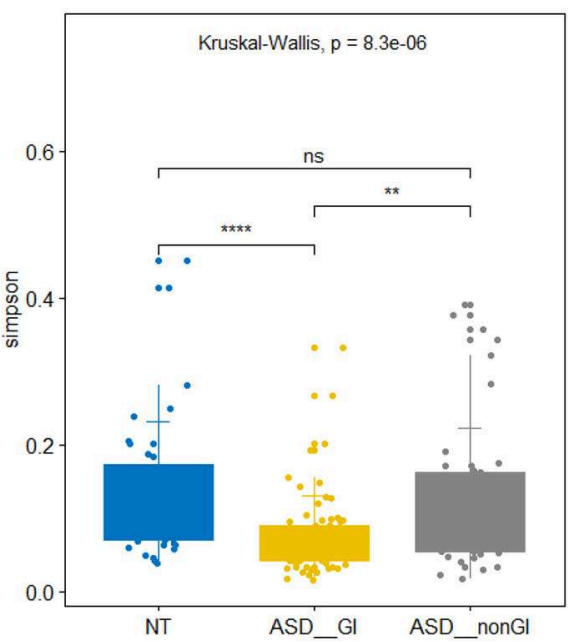

FIGURE 1 | Box plots of $\alpha$ diversity of intestinal flora. (A) Shannon index. (B) Simpson index. NT represents neurotypical subjects. ASD_Gl and ASD_nonGI represent autistic subjects with and without gastrointestinal symptoms, respectively (Kruskal-Wallis test, $p<0.05$ ). ${ }^{*} P<0.05,{ }^{* \star} P<0.01,{ }^{* * \star *} P<0.001$. 
indicate that probiotics treatment, when applied in combination with ABA training, can alleviate the symptoms in both ASD children with and without GI illness. In $83.8 \%$ of ASD children, the ATEC total scores decreased by 8.1 points in average. Scores for Speech/Language Communication, Sociability, Sensory/Cognitive Awareness and Health/Physical/Behavior also significantly decreased. Among them, Health/Physical/Behavior scores declined the most. 86.7\% (13/15) of ASD children without GI events showed improvement in ATEC scores. Their average $A T E C$ score dropped from 59.3 to 50.2 . The rate of improvement in ATEC score for children with ASD and GI abnormalities was $78.9 \%(17 / 22)$, and their average decrease in ATEC score was from 73.2 to 62.9. The rate of improvement in the ASD group without GI events was higher than that in the ASD group with GI abnormalities. ATEC scores showed no significant change in the control group (Table 3). Our results indicate that autistic children without GI symptoms are sensitive to probiotics treatment.

A gastrointestinal questionnaire was used to evaluate the ASD group with GI symptoms. In the treatment group, 19 out of 22 (86.4\%) children have their GI score decreased. The mean GI score in the 19 cases decreased from 2.26 to 0.84 . Scores in 3 cases

TABLE 2 | Bacterial abundance at the level of phylum and genus in ASD and NT groups (Wilcoxon rank test $p$-value and $q$-value $<0.05$ ).

\begin{tabular}{lcccc}
\hline & ASD (mean) & NT (mean) & $\boldsymbol{p}$-value & $\boldsymbol{q}$-value \\
\hline PHYLUM LEVEL & & & & \\
p_Bacteroidetes & 0.12911 & 0.24565 & $7.15 \mathrm{E}-06$ & $2.86 \mathrm{E}-05$ \\
p_Firmicutes & 0.39346 & 0.59019 & 0.028254 & 0.064581 \\
p_Actinobacteria & 0.04222 & 0.07014 & $5.62 \mathrm{E}-05$ & 0.00018 \\
p_Proteobacteria & 0.02626 & 0.04504 & $6.84 \mathrm{E}-12$ & $1.09 \mathrm{E}-10$ \\
GENUS LEVEL & & & & \\
g_Bacteroides & 0.07772 & 0.18368 & $2.21 \mathrm{E}-07$ & $2.13 \mathrm{E}-05$ \\
g_Bifidobacterium & 0.03946 & 0.06787 & $3.66 \mathrm{E}-05$ & 0.000813 \\
g_Ruminococcus & 0.01865 & 0.04330 & $7.77 \mathrm{E}-05$ & 0.001536 \\
g_Lachnospira & 0.01844 & 0.05337 & $2.39 \mathrm{E}-07$ & $2.13 \mathrm{E}-05$ \\
g_Roseburia & 0.02674 & 0.05834 & $1.18 \mathrm{E}-06$ & $5.25 \mathrm{E}-05$ \\
g_Blautia & 0.01084 & 0.02157 & 0.000492 & 0.006256 \\
\hline
\end{tabular}

remained unchanged. No significant improvements in GI score were found in the 11 children in the control group (Table 3 ).

We collected the observational questionnaire about children with ASD before and after probiotics treatment from parents. Among the 31 children who showed decreased ATEC scores, 17 also showed improvement in their behavioral symptoms (eye contact, obedience, self-injury behavior, etc.), 18 showed mood improvement (crying frequency, compliance), 19 patients showed improvement in eating-related symptoms (Improvement in appetite and pickiness), and 13 patients showed improvements in sleep quality. The behavioral and emotional improvement of ASD children with GI events was more pronounced than in children without GI events. ASD children without GI events showed more pronounced improvement in sleep quality and eating habits (Table 3).

During probiotics treatment, 2 patients developed diarrhea $(2 / 37) 1$ week after taking probiotics; the adverse reactions disappeared after the third week of continuous use. This may be because of the Jarisch-Herxheimer die-off reaction. No serious adverse reactions such as infection or exacerbation of symptoms occurred during the study. This demonstrates that a dosage of $30-40$ billion CFU per day is suitable in this study.

\section{DISCUSSION}

Previous studies did not include participants of non-Western ethnicities and concentrated on ASD children with GI events. In this study, we recruited ASD children with and without GI symptoms from multiple centers in China. In this way, our research has a wide sample and better represents children with ASD. The ratio of male to female in our recruits was 1:1 in the NT group and 5:1 in the ASD group. The reason we think is that the incidence of ASD in boys is higher than girls, Early studies showed that autism affects 45 times more males than females (25). Our results indicate that there is a significant difference in the intestinal microbiota between ASD children and NT children regardless of their gastrointestinal problems. The abundance of beneficial bacteria in children with ASD was here found to be significantly lower than in non-ASD children. The abundance of Bacteroidetes

TABLE 3 | Changes in ATEC and GI scores before and after 4-week probiotics treatment.

\begin{tabular}{|c|c|c|c|c|}
\hline Group (N) & Probiotics (37) & Probiotics NGI (15) & Probiotics GI (22) & Control (28) \\
\hline ATEC decrease (\%) & $83.8 \%$ & $86.7 \%$ & $78.9 \%$ & $53.6 \%$ \\
\hline ATEC total (mean) & $67.1-59.0\left(7.2 \times 10^{-7}\right)$ & $59.3-50.2(0.0013)$ & $73.2-62.9\left(1.7 \times 10^{-4}\right)$ & $59.8-56.8(0.89)$ \\
\hline ATEC Speech/Language Communication (mean) & $14.6-13.2\left(1.9 \times 10^{-5}\right)$ & $13.4-12.2(0.014)$ & $15.9-14.4\left(3.6 \times 10^{-4}\right)$ & $11.5-11.1(0.93)$ \\
\hline ATEC Sociability (mean) & $16.6-14.3\left(6.4 \times 10^{-5}\right)$ & $14.9-12(0.0057)$ & $15.9-15.1(0.0046)$ & $15.8-14.2(0.11)$ \\
\hline ATEC Sensory/Cognitive Awareness (mean) & $18.1-16.4(0.0029)$ & $15.8-14.1(0.023)$ & $20.0-17.4(0.023)$ & $16.1-15.9(1)$ \\
\hline ATEC Health/Physical/Behavior (mean) & $17.8-14.2\left(1.2 \times 10^{-4}\right)$ & $15.2-11.8(0.031)$ & $19.7-15.9(0.0016)$ & $16.4-16.3(0.83)$ \\
\hline Gl score decrease (\%) & - & - & $86.4 \%$ & - \\
\hline Gl score decrease (mean) & - & - & $2.26-0.84\left(1.6 \times 10^{-4}\right)$ & - \\
\hline
\end{tabular}

N represents the total number of subjects in each group. The left and right sides of the "-" are the scores before and after probiotics treatment. The numbers in parentheses are $p$-values. GI and NGI indicate the presence and absence of Gl symptoms, respectively. 
in the ASD group was significantly lower than in the NT group, while the ratio of Firmicutes abundance to Bacteroidetes abundance was significantly higher than in the NT group. This suggests that although some ASD children do not exhibit gastrointestinal problems, their intestinal microbiota are, nonetheless, significantly different from those of nonASD individuals.

Several studies and clinical trials have been conducted on the treatment of ASD with probiotics in attempt to reconstitute intestinal microbiota (26-28). However, they have been limited to participants of Western ethnicity. Some studies, despite better design than the above studies, have not drawn definite conclusions (29). Our study is the first report of probiotics treatment for Chinese ASD children. We have shown that after 4 weeks of treatment, more than $80 \%$ of ASD children's ATEC scores decreased. To our surprise, ASD children both with and without GI symptoms were found to be sensitive to probiotics treatment. In addition, a greater proportion of ASD children without GI symptoms responded to probiotics treatment than ASD children with GI symptoms. This is probably because ASD children without GI symptoms have less abnormal intestinal microbiota than ASD children with GI and are therefore more sensitive to probiotics treatment. Previous reports have shown that GI symptoms are correlated with the severity of ASD (15) ASD with GI may require longer treatment and higher doses of probiotics. Our results also show a significant decrease in the ATEC score in the probiotics group relative to the control group, which indicates that the probiotics can increase the intervention effect of ABA training on the child.

The strengths of our study are obvious. First of all, it is a multicenter clinical study of Chinese children. Secondly, the specimens we collected were children's feces, and there were no invasive and injurious operations on children. Thirdly, our findings include the results of probiotic intervention in some children. However, our study also has a few limitations. Our sample size is small, the duration of probiotics treatment was relatively short, and the study was not blinded. Future studies should be performed with larger sample sizes, longer treatment time, longer followup time, and more assessment parameters. Our research can be blinded by giving the ABA only group a pill placebo instead of a probiotic.

\section{REFERENCES}

1. Wang F, Lu L, Wang SB, Zhang L, Ng CH, Ungvari GS, et al. The prevalence of autism spectrum disorders in China: a comprehensive meta-analysis. Int J Biol Sci. (2018) 14:717-25. doi: 10.7150/ijbs.24063

2. Kim YS, Leventhal BL. Genetic epidemiology and insights into interactive genetic and environmental effects in autism spectrum disorders. Biol Psychiatry. (2015) 77:66-74. doi: 10.1016/j.biopsych.2014.11.001

3. Fowlie G, Cohen N, Ming X. The perturbance of microbiome and gut-brain axis in autism spectrum disorders. Int J Mol Sci. 19:E2251. doi: 10.3390/ijms19082251

4. Lacorte E, Gervasi G, Bacigalupo I, Vanacore N, Raucci U, Parisi P. A systematic review of the microbiome in children with neurodevelopmental disorders. Front Neurol. 10:727. doi: 10.3389/fneur.2019.00727

\section{CONCLUSIONS}

Intestinal microbiota is different between the normal children and the ASD children with or without GI. Probiotics treatment can reduce the magnitude $\mathrm{ASD}$-related and gastrointestinal symptoms. In combination with $\mathrm{ABA}$ training, probiotics treatment can bring benefits to ASD children with and without GI. Clinical trials with a more rigorous design and larger sample size are needed for validation.

\section{DATA AVAILABILITY STATEMENT}

Publicly available datasets were analyzed in this study. This data can be found here: http://www.chictr.org.cn.

\section{ETHICS STATEMENT}

The studies involving human participants were reviewed and approved by Ethics Committee for Biomedical Research, First Hospital of Peking University. Written informed consent to participate in this study was provided by the participants' legal guardian/next of kin.

\section{AUTHOR CONTRIBUTIONS}

YH: full access to all the data in the study and takes responsibility for the integrity of the data and the accuracy of the data analysis. MN, YH, SZ, and QL: concept and design. All authors: acquisition, analysis, or interpretation of data and critical revision of the manuscript for important intellectual content. $\mathrm{MN}$ and $\mathrm{YH}$ : drafting of the manuscript. $\mathrm{MN}$ and $\mathrm{SZ}$ : statistical analysis. YH: obtained funding. JZ, FW, WD, GD, HL, WR, PY, CG, HT, XG, and SZ: administrative, technical, or material support.

\section{FUNDING}

This study was supported by Beijing Natural Science Foundation (S170003). This work was also supported by National Key Research and Development Program of China (No. 2016YFC1306201) and Beijing Key Laboratory of Molecular Diagnosis and Study on Pediatric Genetic Diseases (No. Z0317).

5. Sharon G, Cruz NJ, Kang DW, Gandal MJ, Wang B, Kim YM, et al. Human gut microbiota from autism spectrum disorder promote behavioral symptoms in mice. Cell. (2019) 177:1600-18 e17. doi: 10.1016/j.cell.2019.05.004

6. Srikantha P, Mohajeri MH. The possible role of the microbiotagut-brain-axis in autism spectrum disorder. Int J Mol Sci. 20:E2115. doi: 10.3390/ijms20092115

7. Iannone LF, Preda A, Blottiere HM, Clarke G, Albani D, Belcastro V, et al. Microbiota-gut brain axis involvement in neuropsychiatric disorders. Expert Rev Neurother. (2019) 19:1037-50. doi: 10.1080/14737175.2019.16 38763

8. Chaidez V, Hansen RL, Hertz-Picciotto I. Gastrointestinal problems in children with autism, developmental delays or typical development. J Autism Dev Disord. (2014) 44:1117-27. doi: 10.1007/s10803-0131973-x 
9. Strati F, Cavalieri D, Albanese D, De Felice C, Donati C, Hayek J, et al. New evidences on the altered gut microbiota in autism spectrum disorders. Microbiome. 5: 24. doi: 10.1186/s40168-017-0242-1

10. Ding HT, Taur Y, Walkup JT. Gut microbiota and autism: key concepts and findings. J Autism Dev Disord. (2017) 47:480-9. doi: 10.1007/s10803-016-2960-9

11. $\mathrm{Xu} \mathrm{M}, \mathrm{Xu} \mathrm{X}, \mathrm{Li}$ J, Li F. Association between gut microbiota and autism spectrum disorder: a systematic review and meta-analysis. Front Psychiatry. 10:473. doi: 10.3389/fpsyt.2019.00473

12. Martinez-Gonzalez AE, Andreo-Martinez P. The role of gut microbiota in gastrointestinal symptoms of children with ASD. Medicina. 55:E408. doi: $10.3390 /$ medicina55080408

13. Adams JB, Johansen LJ, Powell LD, Quig D, Rubin RA. Gastrointestinal flora and gastrointestinal status in children with autism-comparisons to typical children and correlation with autism severity. BMC Gastroenterol. 11: 22. doi: 10.1186/1471-230X-11-22

14. Buffington SA, Di Prisco GV, Auchtung TA, Ajami NJ, Petrosino JF, Costa-Mattioli M. Microbial reconstitution reverses maternal dietinduced social and synaptic deficits in offspring. Cell. (2016) 165:1762-75. doi: 10.1016/j.cell.2016.06.001

15. Tomova A, Husarova V, Lakatosova S, Bakos J, Vlkova B, Babinska K, et al. Gastrointestinal microbiota in children with autism in Slovakia. Physiol Behav. (2015) 138:179-87. doi: 10.1016/j.physbeh.2014.10.033

16. Grossi E, Melli S, Dunca D, Terruzzi V. Unexpected improvement in core autism spectrum disorder symptoms after long-term treatment with probiotics. SAGE Open Med Case Rep. (2016) 4:2050313X16666231. doi: 10.1177/2050313X16666231

17. Ng QX, Loke W, Venkatanarayanan N, Lim DY, Soh AYS, Yeo WS. A systematic review of the role of prebiotics and probiotics in autism spectrum disorders. Medicina. 55:E129. doi: 10.3390/medicina55050129

18. Li Q, Han Y, Dy ABC, Hagerman RJ. The gut microbiota and autism spectrum disorders. Front Cell Neurosci. 11:120. doi: 10.3389/fncel.2017.00120

19. Gilbert JA, Krajmalnik-Brown R, Porazinska DL, Weiss SJ, Knight R. Toward effective probiotics for autism and other neurodevelopmental disorders. Cell. (2013) 155:1446-8. doi: 10.1016/j.cell.2013.11.035

20. Liu YW, Liong MT, Chung YE, Huang HY, Peng WS, Cheng YF, et al. Effects of Lactobacillus plantarum PS128 on children with autism spectrum disorder in Taiwan: a randomized, double-blind, placebo-controlled trial. Nutrients. (2019) 11:E820. doi: 10.3390/nu11040820
21. Ma B, Liang J, Dai M, Wang J, Luo J, Zhang Z, et al. Altered gut microbiota in chinese children with autism spectrum disorders. Front Cell Infect Microbiol. (2019) 9:40. doi: 10.3389/fcimb.2019.00040

22. Liu F, Li J, Wu F, Zheng H, Peng Q, Zhou H. Altered composition and function of intestinal microbiota in autism spectrum disorders: a systematic review. Transl Psychiatry. (2019) 9:43. doi: 10.1038/s41398-019-0389-6

23. First MB. Diagnostic and statistical manual of mental disorders, 5th edition, and clinical utility. J Nerv Ment Dis. (2013) 201:727-9. doi: 10.1097/NMD.0b013e3182a2168a

24. Hall M, Beiko RG. 16S rRNA gene analysis with QIIME2. Methods Mol Biol. (2018) 1849:113-29. doi: 10.1007/978-1-4939-8728-3_8

25. Lai MC, Lombardo MV, Baron-Cohen S. Autism. Lancet. (2014) 383:896-910. doi: 10.1016/S0140-6736(13)61539-1

26. Tabbers MM, de Milliano I, Roseboom MG, Benninga MA. Is Bifidobacterium breve effective in the treatment of childhood constipation? Results from a pilot study. Nutr J. 10:19. doi: 10.1186/1475-2891-10-19

27. Shaaban SY, El Gendy YG, Mehanna NS, El-Senousy WM, El-Feki HSA, Saad K, et al. The role of probiotics in children with autism spectrum disorder: a prospective, open-label study. Nutr Neurosci. (2018) 21:676-81. doi: 10.1080/1028415X.2017.1347746

28. Critchfield JW, van Hemert S, Ash M, Mulder L, Ashwood P. The potential role of probiotics in the management of childhood autism spectrum disorders. Gastroenterol Res Pract. (2011) 2011:161358. doi: 10.1155/2011/161358

29. Santocchi E, Guiducci L, Fulceri F, Billeci L, Buzzigoli E, Apicella F, et al. Gut to brain interaction in Autism Spectrum Disorders: a randomized controlled trial on the role of probiotics on clinical, biochemical and neurophysiological parameters. BMC Psychiatry. (2016) 16:183. doi: 10.1186/s12888-016-0887-5

Conflict of Interest: The authors declare that the research was conducted in the absence of any commercial or financial relationships that could be construed as a potential conflict of interest.

Copyright (C) 2019 Niu, Li, Zhang, Wen, Dang, Duan, Li, Ruan, Yang, Guan, Tian, Gao, Zhang, Yuan and Han. This is an open-access article distributed under the terms of the Creative Commons Attribution License (CC BY). The use, distribution or reproduction in other forums is permitted, provided the original author(s) and the copyright owner(s) are credited and that the original publication in this journal is cited, in accordance with accepted academic practice. No use, distribution or reproduction is permitted which does not comply with these terms. 